\title{
ROUSSEAU ET LE DROIT NATUREL ${ }^{1}$
}

Yves VARGAS ${ }^{2}$

- RESUMÉ : C'est une erreur d'atribbuer à Rousseau une position favorable au droit naturel, la lecture attentive des textes explique la forme complexe mais radicale du refus chez cet auteur de faire la moindre concession à ce concept.

- MOTS-CLÉS : Rousseau, Droit Naturel, Positivisme, Loi Naturelle.

Je vais examiner si la théorie politique de Jean-Jacques Rousseau accepte le Droit naturel ou si elle le rejette. C'est une question très classique, souvent évoquée : Jean-Jacques Rousseau est-il jusnaturaliste ou juspositiviste? Le fondement du droit chez Rousseau précède-t-il les conventions humaines, ou bien le droit n'est-il rien d'autre qu'une construction humaine ? Or, si cette question - naturalisme ou positivisme - est classique, on peut dire, par contre, qu'elle n'est pas classée, n'ayant pas reçu une réponse qui aurait mis fin aux discussions. Les penseurs, les philosophes qui lisent et relisent Rousseau trouvent, à chaque fois, de nouvelles interprétations, de nouvelles nuances, et ouvrent de nouvelles perspectives.

Pour ma part, je voudrais analyser sobrement la question, c'est-à-dire que je vais tenter de lire quelques textes de Rousseau à propos du Droit naturel et de la Loi naturelle, pour voir ce qu'il dit ou ce qu'il ne dit pas. Non pour apporter une nouvelle interprétation mais pour dresser un constat.

1 Confèrence Prononcée à l'Université Catholique de São Paulo - PUC-SP.

2 Présidente du Groupe d'Etudes du Matérialisme Rationnel, membre de la Fondation Gabriel Péri. Essai reçu à 05/08 et approuvé à 06/08. 
Dès la fin du $17^{\text {ème }}$ siècle et durant tout le siècle suivant, les théories du Droit naturel dominent la pensée philosophique et juridique. L'idée qu'il existe des lois qui précèdent et surplombent toutes les juridictions particulières est, au $18^{\text {ème }}$ siècle une évidence, un lieu commun qu'on ne se donne même pas la peine de la rappeler. Par exemple, l'éditeur de J.-J. Burlamaqui préface le livre du juriste par ces mots tranquilles :

Je pensais que le Droit naturel devait précéder le Droit civil, qu'il fallait étudier le droit immuable avant le droit arbitraire. (Burlamaqui, 1989, préface)

Cette idée de Droit naturel n'est pas une mode, un caprice de salon, c'est un système qui enveloppe des principes moraux et anthropologiques, des enjeux politiques : dire qu'il existe une loi avant la loi et au-dessus des lois, n'est pas un discours innocent ; opposer la loi des princes et des Etats à la Loi naturelle en taxant la première d' "arbitraire " et l'autre d' " immuable " n'est pas rien, et c'est même une idée politique subversive qui peut aboutir à l'insurrection ou à la désobéissance. ${ }^{3}$

Il est utile de rappeler en deux mots à quoi ressemble cette évidence.

Le Droit naturel est un droit, il porte sur la question des rapports entre les hommes de façon à distribuer l'interdit et le licite, sur la base du juste et de l'injuste, et, bien entendu, c'est un droit en ce qu'il sanctionne les infractions. « La loi de la nature serait vaine si personne, dans l'état de nature, n'avait le pouvoir d'en assurer l'exécution pour protéger les innocents et imposer la retenue aux délinquants » (Locke, 1992, § 7).

Afin que la loi puisse produire cet effet [...], il est nécessaire qu'elle soit [...] accompagnée d'une sanction convenable. (Burlamaqui, 1989, I, ch.4)

Le caractère relationnel et contraignant de cette Loi naturelle apparaît dans son principe fondateur qui est le respect des contrats auxquels on a souscrit, condition juridique qui ouvre la voie :

Le Droit naturel [...] défend de manquer à sa parole, [...] il ordonne d'être fidèle à ses engagements. (ibidem)

Cette loi est antérieure et supérieure à la loi positive. Cela est général chez tous les auteurs qui souscrivent à la théorie du Droit naturel.

3 J. Locke nomme cette ouverture hors la loi au nom de la Loi naturelle : l'appel au ciel, c'est-à-dire le retour à l'état pré-politique : «Il existe une loi antérieure à toutes les lois positives des hommes, qui lui réserve [au peuple] la décision ultime [...] qui consiste à [...] en appeler au ciel » (Locke, 1992, § 168). Voir aussi Grotius, 1984 (II, ch.26), qui traite de la désobéissance. 
Concernant Rousseau, on sait qu'il ne reconnait pas la légitimité des gouvernements en place, qu'il les qualifie de "tyranniques" ou "despotiques ", ce qui laisse à penser qu'il existe pour lui des lois supérieures aux lois positives. Mais d'un autre côté, il affirme que la loi décrétée par chaque peuple est la seule loi fondamentale, ce qui laisse à penser que chaque peuple peut se donner la loi qu'il veut sans qu'aucune loi supérieure puisse intervenir. On imagine alors Rousseau, adversaire des rois et favorable à la tyrannie des peuples, Rousseau fondateur du "totalitarisme ", en songeant à certaines dictatures révolutionnaires. Pour soustraire Rousseau à ces accusations, quelques brillants auteurs se sont attachés à trouver, au fond de sa pensée, des symptômes de son attachement au Droit naturel, ce qui permet à la fois de le rattacher à son siècle et de lui donner une figure plus agréable.

Pour envisager cette question, je vais d'abord résumer ce que Rousseau dit du Droit naturel dans ses principaux traités : le Discours sur l'origine de l'inégalité, l'article Economie politique, le manuscrit de Genève, et $\mathrm{Du}$ Contrat social. J'examinerai ensuite quelques points délicats, concernant ses formules et la cohérence de son système.

\section{Le Droit naturel et la Loi naturelle dans les traités de Rousseau}

\section{Le Discours sur l'origine de l'inégalité}

Dans la préface de son Discours sur l'origine de l'inégalité, Rousseau se livre à une analyse serrée de la notion de Loi naturelle afin de l'écarter. Il faut rappeler que le sujet proposé par l'académie de Dijon était : Quelle est l'origine de l'inégalité parmi les hommes et si elle est autorisée par la Loi naturelle? Par sa préface, Rousseau annule la deuxième partie de la question.

Dans un premier temps, il remarque que les théoriciens du Droit naturel ne sont pas d'accord sur les principes de ce droit, puis il examine la question en distinguant, après Montesquieu, deux sens. Premier sens, la Loi naturelle comprise comme déterminisme naturel : " expression des rapports généraux établis par la nature entre tous les êtres animés ". Second sens, la loi au sens juridique, qui impose une règle de conduite qu'on peut suivre ou ne pas suivre: "règle prescrite à un être moral, c'est-à-dire intelligent ", mais une telle loi ne saurait être individuelle, elle concerne l'homme socialisé, "dans ses rapports avec d'autres êtres ». 
Cela posé, et sans se prononcer encore sur l'existence d'une telle loi, il réfute l'argument par le bonheur. Les jusnaturalistes font la démonstration suivante : les hommes cherchent naturellement le bonheur, or la loi permet de vivre heureux, elle est donc fondée sur la nature. Rousseau déclare que cette démonstration ne vaut rien, car le fait que les lois humaines sont utiles aux hommes ne prouve en rien qu'elles soient naturelles, des conventions arbitraires peuvent être tout aussi utiles, le bonheur peut venir de la nature comme il peut venir de l'artifice :

On donne le nom de Loi naturelle à la collation des ces règles sans autre preuve que le bien qu'on trouve qui résulte de leur pratique universelle. Voilà [...] une manière très commode [...] d'expliquer la nature des choses par des convenances presque arbitraires.

L'autre réfutation est d'ordre anthropologique : la Loi naturelle ne peut être connue que pour autant que l'homme soit doué de raison. Or, la raison n'est pas un don immédiat de la nature, mais elle se forme dans le rapport social. Si la connaissance de la Loi naturelle suppose la société, la société ne peut donc être fondée sur elle.

Il est impossible d'entendre la loi de la nature sans être un grand raisonneur et un profond métaphysicien. Les hommes ont dû employer pour l'établissement de la société des lumières qui ne se développeront que [...] dans la société même.

Rousseau oppose donc à l'idée de Loi naturelle une triple critique :

1/ Critique épistémologique : il y a loi et loi, on ne doit pas inférer l'existence d'une juridiction naturelle à partir de l'ordre objectif du monde ;

2/ Critique méthodologique : on ne peut déduire la naturalité des lois à partir de leur utilité universelle ;

3/ Critique anthropologique: l'homme capable de connaître la Loi naturelle qui fonde la société doit être déjà social.

On peut penser, à partir de la troisième critique, que Rousseau accepte l'idée d'une Loi naturelle à retardement, une loi qui attendrait que l'homme fût assez développé pour qu'il pût enfin reconnaître, après-coup, le vrai principe de son état. L'arbre de la société humaine pousserait par le milieu du tronc et retrouverait ensuite ses racines. On verra, au cours des lectures suivante si cet espoir est permis.

Après cette mise à l'écart, Rousseau parle de l'amour de soi et de la pitié, sortes d'instincts qui ne nécessitent pas d'intelligence, et à partir desquels devraient être fondées « toutes les règles du Droit naturel ». Ce passa- 
ge a été l'objet d'interprétations favorables au Droit naturel, un Droit naturel modifié mais bien réel. Je cite le passage :

Deux principes antérieurs à la raison, dont l'un nous intéresse ardemment à notre bien-être et à la conservation de nous-mêmes, et l'autre nous inspire une répugnance naturelle à voir périr ou souffrir tout être sensible et principalement nos semblables. C'est du concours et de la combinaison de ces deux principes [...] que me paraissent découler toutes les règles du Droit naturel.

Notons que Rousseau parle ici du «Droit naturel » et non plus, comme précédemment de la « Loi naturelle ». Il ne dit pas que l'amour de soi et la pitié fondent la Loi naturelle mais le Droit naturel. Il semble qu'il prenne en compte la distinction proposée par Hobbes entre ces deux notions. Hobbes oppose le Loi naturelle qui détermine des règles et des interdictions, et le Droit naturel qui n'est rien d'autre que la force et le désir, c'est-à-dire le droit de faire tout ce qui nous plait dans les seules limites de notre force. Le Droit naturel, chez Hobbes aboutit à l'état de guerre auquel met fin un contrat produit par l'intelligence humaine qui « calcule » son avantage à renoncer au droit sur toute chose et à respecter une loi. Ainsi l'homme hobbesien, foncièrement méchant devient social par calcul, par raison. A cette théorie, les philosophes du Droit naturel ont riposté en introduisant au sein du droit primitif sur toute chose un élément qui le modère et le limite, la sociabilité, une tendance à rechercher les rapports humains, et donc à limiter cette brutalité originelle.

Rousseau répond ici à ces deux théories à la fois, celle de la méchanceté originelle et celle de la sociabilité. A Hobbes il accorde que le Droit naturel est bien fondé sur une recherche égoïste, un désir de bien-être qui ne se soucie pas d'autrui. Mais ayant refusé la raison à l'homme originel, Rousseau se trouve alors en situation difficile : comment, sans le " calcul » mettre fin à la guerre générale que ces chocs entre égoïsmes aveugles engendreront ? Il ajoute donc la pitié, sorte de frein instinctif. La pitié plutôt que la sociabilité car la pitié n'est pas sociable, elle n'est pas un rapport aux autres mais un rapport de moi à moi, un déplaisir qui vient gâcher mon plaisir quand mon plaisir fait souffrir un être vivant, animal ou homme. Ces règles du Droit naturel ne sont donc en rien des règles qui fondent la société, elles restent engluées dans la sauvagerie solitaire, elles ne sont pas des règles qui se présentent à un être raisonnable qui peut les accepter ou les refuser, bref elles n'ont aucun des caractères requis pour parler de Loi naturelle, qui, il faut le rappeler, supposent qu'on a affaire à des êtres libres et intelligents. Ces principes du Droit naturel, blottis au fond de la nature humaine dès son origine, pourront, en se développant, donner les piliers de la 
morale, mais en aucun cas de la politique, à moins de vouloir confondre ces deux domaines, ce que Rousseau ne fait pas : on y reviendra.

Notons que la suite du texte qui décrit la genèse de l'humanité ne fait aucun recours à l'idée de Loi naturelle. Dans la période de « jeunesse du monde ", Rousseau explique que la jalousie sexuelle fait couler le sang humain, et il ne parle pas de Loi naturelle sur ce point, par exemple.

\section{Economie politique}

En deux points, au sein du même paragraphe, l'article Economie politique soutient des positions qu'on peut qualifier sans hésiter de "positivisme juridique ». D'une part il pose que la justice est une affaire d'Etat, elle ne transcende pas le corps politique et chaque peuple (c'est-àdire chaque volonté générale) décide de sa propre justice sans qu'il soit possible d'en juger :

Cette volonté générale [...] est pour tous les membres de l'Etat [...] la règle du juste et de l'injuste, vérité qui [...] montre avec combien de sens tant d'écrivains ont traité de vol la subtilité prescrite aux enfants de Lacédémone, pour gagner un frugal repas ; comme si tout ce qu'ordonne la loi pouvait ne pas être légitime. (Rousseau, 1964a, p.245)

Si la loi de l'Etat est le dernier mot de la justice, cela annule l'idée d'une justice universelle, la justice s'arrête aux frontières de l'Etat et peut changer selon les choix du peuple voisin. L'idée d'un "droit des gens » transnational, idée chère aux théories du Droit naturel, est inacceptable.

Cette règle de justice, sûre par rapport à tous les citoyens, peut être fautive avec les étrangers [...]. C'est qu'alors la volonté de l'Etat, quoique générale par rapports à ses membres, ne l'est plus par rapport aux autres Etats et à leurs membres.

Jusque là il n'y a pas de discussion possible, le rejet du Droit naturel et l'inscription dans le positivisme le plus strict saute aux yeux, mais la suite immédiate du texte semble renverser cette situation en son contraire, et introduit une «Loi de nature ». Je cite donc la suite immédiate du texte :

C'est qu'alors la volonté de l'Etat [...] ne l'est plus par rapport aux autres Etats et à leurs membres, mais devient pour eux une volonté particulière et individuelle qui a sa règle de justice dans la Loi de nature [...] car alors la grande ville du monde devient un corps politique dont la loi de nature est toujours la volonté générale et dont les Etats et les divers peuples ne sont que les membres individuels. 
S'agit-il d'un retournement, d'une contradiction, d'une concession faite à Diderot qui dirige l'Encyclopédie dans laquelle cet article «Economie politique » doit prendre place ? Ces hypothèses extérieures sont toutes acceptables et avisées, mais je crois qu'il faut d'abord examiner le texte luimême avec attention. Or, la lecture attentive du passage montre que cette "Loi de nature " est une fiction, et même plus, une fiction positiviste. En effet, cette «Loi » n'est rien d'autre que la volonté générale des Etats réunis, et on a vu que chaque Etat n'est rien d'autre qu'une volonté générale particulière, ce qui signifie que la volonté générale de tous les Etats réunis dépend de ces Etats, qu'elle ne s'impose pas à eux d'en haut, comme une vérité préalable : si on change les Etats, s'ils changent leurs lois, la volonté générale changera aussi ; si tous les Etats réunis décident que les enfants voleront leur repas la « Loi naturelle » dictera que cela n'est pas un vol. Cette Loi est postérieure à la loi positive et en dépend; si elle est dite " naturelle " (sans doute la concession à Diderot porte sur le mot et seulement sur le mot), c'est parce qu'elle couvre la planète: on devrait dire qu'une volonté générale de tous les Etats et de tous les peuples serait la volonté générale " maximale ", plutôt que " naturelle ». En effet, dans les pages précédentes, Rousseau explique qu'à chaque niveau de la réalité sociale il existe une volonté générale plus ou moins grande qui concerne chaque corps social : une famille, un métier, une religion, une équipe, et même une association de brigands, ont une volonté générale ; mais par rapport à une volonté générale plus grande, elle devient particulière : par emboîtements successifs de volontés générales de plus en plus en plus grandes mais toujours conventionnelles, on en arrive à l'Etat qui est une réalité, puis en poursuivant on arrive à la " ville du monde ", qui est une fiction théorique, et qui engloberait toutes les précédentes et assureraient la même loi à tous ses citoyens, (c'est-à-dire à toute l'humanité, puisqu'il n'y aurait plus aucun étranger sur terre). Ainsi s'établirait une justice universellement reconnue. Justice conventionnelle entre Etats et peuples qu'il nomme, sans raison théorique, la "Loi de nature ». Si on doute que cette "Loi de nature " n'est que l'extension des emboîtements de volontés générales positives, il n'est qu'à lire la conclusion de Rousseau sur cette question de la "ville du monde ", il écrit: "ce qui rentre également dans les principes établis", c'est-à-dire des principes qui partent des petites volonté générale jusqu'aux grandes. Ainsi Rousseau réalise ce tour de force de donner au concept de Loi naturelle une signification positiviste.

\section{Manuscrit de Genève ( $\mathrm{Du}$ Contrat social $1^{\text {ère }}$ version)}

Le manuscrit de Genève est le premier brouillon du Contrat social. Il contient quelques pages sur le problème du Droit naturel, pages qui ont été abandonnées dans le texte final. 
Ainsi, plusieurs lecteurs y ont vu une hésitation de Rousseau sur ce point, on a même pu construire une théorie implicite de Rousseau sur une notion qui se trouve dans ce texte, celle de "Droit naturel raisonné ». En fait, la disparition de cette notion est liée à l'abandon d'une première problématique, celle de l'intérêt. Dans le manuscrit de Genève, Rousseau s'efforce de construire l'idée de peuple et de droit politique, à partir de la notion anthropologique d'intérêt: comment l'individu peut-il voir que son intérêt personnel est d'opter pour l'intérêt général ? Il examine alors si la Loi naturelle serait de nature à l'éclairer, et c'est dans cette perspective qu'il développe son analyse. Dans le texte définitif, il abandonne cette question de l'intérêt, et du même coup, la considération de la Loi naturelle n'est plus nécessaire.

L'analyse de la Loi naturelle s'établit en cinq étapes, je les annonce en deux mots, puis je les précise un peu, et je serai amené à développer la cinquième. Voici les étapes :

1. Hypothèse : on suppose que la Loi naturelle existe et que le genre humain est unifié. Quand la loi pourra être connue (par la raison), elle sera annulée (par les passions) ;

2. Supposons qu'elle soit connue par la raison. Elle ne sera pas appliquée car elle ne présente aucune garantie ;

3. Retour sur la deuxième hypothèse: la raison peut-elle la connaître ? Non car la raison se mélange avec les préjugés sociaux (les mentalités) et appelle « Loi naturelle » la loi positive modifiée ;

4. Démonstration : il a fallu attendre le développement des mentalités pour que cette idée naisse dans l'histoire (le christianisme) ;

5. Quel fondement peut-on donner à cette idée ? Elle peut être imaginée à partir d'une étude sociologique sur les mœurs sociales et une généralisation par analogie : c'est le Droit naturel raisonné.

Je détaille un peu ces arguments.

Le premier reprend l'hypothèse de la théorie générale du Droit naturel. Il existe une Loi naturelle et les hommes sont regroupés naturellement dans un genre humain capable de prendre des décisions, une sorte de peuple spontané. Selon cette théorie, les hommes formeront un contrat social à partir de cette loi préalable. Rousseau répond que ce contrat est une chimère pour la raison suivante : la connaissance de la loi suppose l'existence de la raison, or la raison ne se développe qu'avec les progrès de la société, et comme ces progrès développent en même temps les passions égoïstes qui aveuglent les hommes, cette loi ne pourra être connue qu'au moment où ils seront incapables de la reconnaître : 
Ajoutons, si l'on veut cette supposition; concevons le genre humain comme une personne morale ayant un sentiment d'existence commun [...] qui fasse agir chaque partie pour une fin générale et relative au tout. Concevons que ce sentiment commun soit celui de l'humanité et que la Loi naturelle soit le principe actif de toute cette marche [...]. Nous trouverons que [...] les notions de Loi naturelle, qu'il faudrait plutôt appeler Loi de raison, ne commencent à se développer que quand le développement antérieur des passions rend impuissants tous ses préceptes. Par où l'on voit que ce prétendu traité social dicté par la nature est une véritable chimère. (Rousseau, 1964b, p.286-7)

Supposons à présent que l'intérêt n'aveugle pas les hommes et qu'ils soient capables de concevoir la Loi naturelle et de l'examiner raisonnablement. Chacun peut constater qu'elle lui serait profitable si tous la respectaient en même temps que lui ; mais voyant qu'elle n'a aucune force pour obliger les autres, il serait déraisonnable d'être le seul à la respecter au détriment de son seul intérêt. Dans le premier cas la raison ne connaît pas la loi, dans le deuxième elle ne l'applique pas.

Tout ce que vous me dites sur les avantages de la loi sociale pourrait être bon, si tandis que je l'observais scrupuleusement envers les autres, j'étais sûr qu'ils l'observeraient envers moi ; mais quelle sûreté pouvez-vous me donner là-dessus ? [...] Il s'agit de me montrer quel intérêt j'ai d'être juste. (ibidem)

Le troisième moment n'est pas anthropologique, il n'oppose plus la raison et les passions, il est d'ordre épistémologique : il s'agit de voir en quoi peut consister cette raison qui serait capable de connaître la Loi naturelle, c'est-à-dire la volonté générale du genre humain. Il s'agirait d'une pure activité de la raison. Rousseau explique alors qu'une telle faculté n'existe pas car le jugement humain est composé d'habitudes, de modèles de pensées, et malgré la bonne foi de chacun, il est impossible de penser dans le vide, toute pensée s'adosse à des expériences. Dans ce cas, la Loi naturelle ne serait qu'une copie involontaire des mentalités juridiques acquises dans les sociétés positives : la raison pure législatrice ne serait qu'une idéologie sociale :

Cette volonté générale [est] dans chaque individu un acte pur de l'entendement qui raisonne dans le silence des passions. [...] Mais où est l'homme qui puisse ainsi se séparer de lui-même ? [...] Combien de fois n'arriverait-il pas à un homme bien intentionné de se tromper sur la règle ? [...] Cette voix [intérieure] n'est formée que par l'habitude de juger et de sentir dans le sein de la société et selon ses lois. (ibidem)

Ce n'est que de l'ordre social établi parmi nous que nous tirons toutes les idées de celui que nous imaginons. Nous concevons la société générale d'après les sociétés particulières [...] et nous ne commençons à devenir hommes qu'après avoir été citoyens. (idem, p.287) 
Ainsi donc, non seulement le contrat social est une chimère mais la Loi naturelle est une bévue. Conséquence : le genre humain unifié n'existe pas à l'origine, l'homme n'est pas l'origine du citoyen, c'est le contraire qui est vrai, l'hypothèse $\mathrm{n}^{\circ} 1$ est annulée: "Nous ne commençons proprement à devenir hommes qu'après avoir été citoyens ».

Quatrième moment, directement déduit de ce qui précède : si la raison illusoirement pure n'est qu'une forme de jugement social, cela signifie que la Loi naturelle a suivi les aléas des mentalités, qu'elle a été produite au fil des préjugés qui se sont succédés dans l'histoire. Après la critique épistémologique, Rousseau entreprend une généalogie historique :

Les saines idées du Droit naturel et de la fraternité commune de tous les hommes se sont répandues assez tard et ont fait des progrès si lents qu'il n'y a que la christianisme qui les ait suffisamment généralisés. (ibidem) ${ }^{4}$

Le cinquième argument porte sur un délicat passage où il est question du « Droit naturel raisonné ». Passage délicat parce qu'il a donné lieu à diverses interprétations profondes et contradictoires. N'étant pas moi-même un "profond métaphysicien ", comme disait Rousseau, je vais me limiter à le lire au ras du texte, en m'efforçant d'oublier ses multiples interprétations.

Le chapitre quatre du livre deux porte en titre : « De la nature des lois et du principe de la justice civile». Rousseau explique que les lois « sont des actes de la volonté générale ", et il poursuit :

Le plus grand avantage qui résulte de cette notion est de nous montrer clairement les vrais fondements de la justice et du droit naturel. En effet, la première loi, la seule véritable loi fondamentale qui découle du pacte social est que chacun préfère en toutes choses le plus grand bien de tous. Or, la spécification des actions qui concourent à ce plus grand bien, par autant de lois particulières est ce qui constitue le droit positif.

Jusque là nous ne sortons pas du droit positif fondateur, c'est-à-dire du positivisme juridique. La "véritable loi fondamentale " ne vient pas de la nature mais bien du " pacte social », elle consiste en un glissement de l'égoïsme vers l'intérêt général ; et tous les actes ordonnés en faveur de cet intérêt général, « la spécification des actions qui concourent à ce plus grand

4 Loin donc que le christianisme soit ici appelé à fonder le Droit naturel comme une vérité universelle et éternelle, il n'est invoqué qu'à titre historique et tardif dans l'histoire humaine. Aller chercher dans les Evangiles un fondement du Droit naturel éternel n'entre pas du tout ici dans la visée de Rousseau, et ce n'est pas en tant que religion vraie qu'il est invoqué mais en tant qu'événement daté et très éloigné de l'origine de l'humanité. Ce texte pourrait tout aussi bien - et aussi mal - être utilisé par des marxistes pour y voir une conception du matérialisme historique. 
bien " sont codifiés, non par la nature qui parle au cœur ou à la raison, mais bien par "des lois particulières », c'est-à-dire « ce qui constitue le droit positif ». Or, le début du texte annonçait qu'on allait voir "clairement les vrais fondements de la justice et Droit naturel $»$ : pour l'instant on n'a rien vu de tel, et le Droit naturel brille par son absence. La suite nous y conduit par l'intermédiaire d'une remarque sur les rapports entre la loi juridique et les manières sociales qui pallient les silences de la loi. Voici :

Tout ce qu'on voit concourir à ce plus grand bien, mais que les lois n'ont point spécifié, constitue les actes de civilité, de bienfaisance, et l'habitude qui nous dispose à pratiquer ces actes à notre préjudice est ce qu'on nomme force ou vertu.

Sachant que c'est de ce passage que Rousseau va bientôt tirer les vrais fondements du Droit naturel, il est justifié de bien le mettre en mémoire. Il y est dit que l'intérêt général, qui est le but que vise la loi, en spécifiant ce qu'on doit faire et ne pas faire pour réaliser cet intérêt, cet intérêt général déborde le cadre strict de la loi, elle ne l'épuise pas, tout n'est pas dit quand la loi a fini de parler. Qu'est-ce que cela veut dire ? Cela veut dire que certains actes qui vont dans le sens de l'intérêt général ne figurent pas dans la spécification des lois, ils ne sont pas rendus obligatoires pas la loi, ce sont les actes de civilité, de bienfaisance. Ces actes, l'entr'aide, la politesse, l'urbanité des conduites, constitue cette spontanéité sociale qui facilite la vie en commun et que Hegel nommera "Slittlichkeit », éthos social, et que Rousseau nomme "vertu » ou " force » parce que l'individu, sans y être forcé, agit coutumièrement pour le bien commun. Notons que cette vertu est un supplétif à la loi, qu'elle présuppose la loi qui donne l'orientation générale, la loi ne consiste pas à codifier la vertu, c'est au contraire la vertu qui consiste à mimer les codifications de la loi hors du champ de la loi. La vertu parle dans le silence des lois et à l'intérieur de l'enceinte des lois. Pardonnez cette insistance, elle est justifiée par ce qui suit : car la suite du texte montre que la Loi naturelle n'est rein d'autre que cette « vertu » élevée au rang d'une généralisation.

Etendez cette maxime à la société générale dont l'Etat nous donne l'idée [...], nous sommes portés à la fois par la nature, par l'habitude et par la raison à en user avec les autres hommes à peu près comme avec nos concitoyens, et de cette disposition réduite en actes naissent les règles du Droit naturel raisonné, différent du Droit naturel proprement dit, qui n'est fondé que sur un sentiment vrai mais très vague et souvent étouffé par l'amour de nous-mêmes.

Voyons donc ce texte en détail. "Etendez cette maxime » : il s'agit de la maxime de la vertu, fondée sur les habitudes d'urbanité, qui vise le plus 
grand bien de tous, en agissant dans le silence des lois positives et dans leur cadre. ${ }^{5}$ A présent, Rousseau propose d'étendre cela, de sortir du cadre politique strict, et de passer d'une société particulière à la "société générale ». Cette "société générale " constituait la "supposition " du début, celle du genre humain unifié en un moi commun, société générale dont il a été montré qu'elle n'est qu'un produit de l'imagination, forgé à partir du modèle des Etats positifs, c'est-à-dire qu'elle n'est pas une réalité. Rousseau propose donc d'étendre une pratique quotidienne sociale à quelque chose qui n'existe pas, qui est une « idée » et rien d'autre, une « idée » qui ne dépasse pas les Etats positifs puisqu'elle en dépend, ce qui est clairement énoncé : "Etendons cette maxime à la société générale dont l'Etat nous donne l'idée ».

L'opération va donc consister à appliquer à l'ensemble du genre humain des conduites de bienveillances qui existent hors des lois, ce qui est concevable puisque, existant hors des lois, on peut supposer qu'elles puissent s'en affranchir totalement et circuler d'un système de lois d'un pays à l'autre, en empruntant les couloirs de silence qui festonnent tous les systèmes de lois: "Nous sommes portés [...] à en user avec les autres hommes comme avec nos concitoyens ». Ainsi donc, par delà les lois, il est possible d'agir pour le plus grand bien de tous, grâce à cette bienveillance étendus de l'Etat à l'humanité toute entière. Il est clair que cette bienveillance a

5 Certains auteurs ont supposé que ces "maximes » sont le principe général de la loi positive, ce principe qui établit que l'intérêt commun passe avant l'intérêt particulier. Le Droit naturel raisonné serait alors la généralisation de la loi positive, le passage de l'Etat politique au monde universel. Deux raisons nous semble s'opposer à cette interprétation. La première tient à la logique des concepts. S'il suffisait de généraliser l'esprit des lois positives, on comprendrait mal l'utilité du développement sur la bienfaisance, la civilité et la vertu : Rousseau n'aurait qu'à déclarer que le Droit naturel vient d'un raisonnement sur le droit positif. Or, un tel développement ne peut aboutir au concept de Droit naturel qu'en devenant indépendant du droit positif, du cadre politique de ce droit : c'est bien cette condition d'indépendance vis-à-vis du droit que montre la vertu, qui conserve bien le principe du droit sans dépendre du droit. En ce sens la vertu est l'intermédiaire nécessaire pour arriver au Droit naturel raisonné. Sinon, ce Droit naturel raisonné ne serait rien d'autre qu'un contrat social mondial à partir des droits positifs particuliers : c'est la figure qu'on trouve dans Economie politique, celle des « degrés » de la volonté générale, et qui n'a besoin ni de la bienfaisance ni du "raisonnement". La deuxième raison est lexicale. Rousseau parle de " maximes ", or ce vocable désigne en général un principe sans obligation, tiré de la conduite humaine, et non des idées codifiées et ordonnées, formulées dans la loi. On peut tirer une maxime à partir d'une conduite mais non à partir de texte explicites. D'ailleurs, chez Rousseau, le terme " maxime " apparaît toujours pour désigner des conduites morales non codifiées mais constatables : le Discours sur l'origine de l'inégalité parle des "maximes de la pitié ", et Emile propose quatre maximes pour formaliser la logique de la pitié. Par ailleurs le législateur ne recourt à aucune maxime pour instituer la loi d'un peuple. Le Droit naturel raisonné, formulé par la raison à partir de " maximes » se réfère donc bien à la vertu, la bienfaisance, et n'est en rien une universalisation des lois positives, qui sont des lois et non des maximes. 
quelques traits ressemblants avec « le droit des gens » cher aux théoriciens du Droit naturel. ${ }^{6}$

Un problème se pose avec évidence : si cette vertu existe à partir des lois et des habitudes inscrites dans telle ou telle société particulière, il est inévitable que cette vertu porte des valeurs, des idéaux, des préjugés, qui appartiennent à la société d'origine et qu'il est impossible de faire circuler d'un bout du monde à l'autre. Rien ne permet de penser que les marges des lois soient plus universelle que les lois elles-mêmes, et Rousseau sait mieux que quiconque l'importance politique de l'opinion publique qui s'insinue partout et qui, loin d'être universelle, façonne chaque peuple et le distingue irrémédiablement de ses voisins. Si on veut donc étendre les principes de la vertu, il faut les vider de leur contenu idéologique, ce que Rousseau confirme : " cette disposition réduite en actes ». La vertu universalisée doit être muette, réduite au spectacle des actes réduits à leurs mouvements, de sorte que le Droit naturel s'établit dans une société imaginaire et un théâtre muet. C'est une construction laborieuse d'inventaire sociologique des comportements aimables détachés de leurs valeurs, et c'est cette construction par généralisation et abstraction que Rousseau nomme le « Droit naturel raisonné »: "De cette disposition réduite en actes naissent les règles du Droit naturel raisonné ".

Le Droit naturel raisonné n'est donc pas la reprise par la raison d'un Droit naturel en attente d'être reconnu et adopté par la raison, il est tout autre chose, la construction méthodique d'un travail de généralisation, d'analogie, d'abstraction, où la raison et l'imagination concourent à fabriquer une idée précise de ce que pourrait être un droit universel, postérieur aux multiples droits positifs et s'étayant sur eux pour les visiter tous. Rousseau propose ici une analyse épistémologique des conditions historiques qui rendent pensables un Droit naturel et des opérations intellectuelles qui peuvent l'édifier. C'est pourquoi, à ce droit naturel fabriqué par la raison, il oppose pour conclure le " Droit naturel proprement dit », c'est-à-dire celui

6 Rousseau donne plusieurs causes hétérogènes qui concourent à cette bienfaisance sans frontières : « la nature, l'habitude et la raison ». La nature n'est pas ici la Loi naturelle, sinon ce laborieux travail d'extension serait inutile, la Loi naturelle est ce qu'il s'agit de comprendre, elle ne peut donc être au principe de sa propre genèse. La nature indique ici très probablement la pitié, la répugnance à faire souffrir, l'habitude suppose la vie sociale, la répétition acquise dans la société politique. Quant à la raison, le texte explique qu'il s'agit de l'assurance de n'être pas soumis à la violence des autres, étant protégés par la loi : on se souvient de ce que répondait l'homme raisonnable et égoïste à l'argument de plus grand bien : si je freine ma violence, qu'est-ce qui me garantit que les autres ne profiteront pas de ma modération contre moi, qu'est-ce qui me garantit de leur violence? Ici, nous sommes sous la garantie des lois et c'est sous cette garantie que peut s'exercer, raisonnablement, la bienfaisance. C'est donc un citoyen protégé par la loi et habitué à la bienveillance sociale qui peut avoir l'idée de cette sorte de droit des gens. 
qui est présenté par les théoriciens du Droit naturel. Ce Droit naturel de la tradition jusnaturaliste, il en salue l'excellence (il dit qu'il est un "sentiment vrai ») et il l'invalide épistémologiquement ("sentiment très vague ») et anthropologiquement (" étouffé par l'amour de nous-mêmes ») :

Droit naturel raisonné, différent du Droit naturel proprement dit, qui n'est fondé que sur un sentiment vrai mais très vague et souvent étouffé par l'amour de nous-même.

Il n'est nullement question de l'affirmation de deux Droits naturels, l'un existant pour soi et inconnaissable, et l'autre qui serait reconnu par la raison après l'établissement des sociétés. Il y a simplement opposition entre deux idées, deux théories, celle du Droit naturel, sentimentale, vague et sans réalité (« étouffé » ne signifie pas en puissance), et celle du Droit naturel raisonné, fiction construite méthodiquement à partir d'observations sociologiques, et qui serait le seul fondement acceptable d'une telle idée. Remarquons qu'en aucun cas ce droit naturel raisonné ne saurait prétendre être au-dessus des lois ou antérieur à elles.

\section{Du Contrat social}

Tout le monde s'accorde à dire que Du Contrat social ne fait aucune place au Droit naturel. Dire qu'il n'y accorde aucune place n'est pas suffisant, on pourrait imaginer qu'il n'en parle pas parce que qu'il s'agit d'une notion si évidente au $18^{\text {ème }}$ siècle qu'il n'est pas nécessaire d'en parler pour y souscrire, c'est pourquoi il y a eu des lecteurs assez attentifs pour en trouver les traces ou les figures renouvelées.

Le premier chapitre annonce : "ce droit ne vient pas de la nature ", il s'agit du "droit sacré » de " l'ordre social », et il conclut : " il est donc fondé sur des conventions ». Les chapitres suivants examinent diverses théories qui expliquent l'ordre social par la soumission aux chefs, et ces trois chapitres présentent une analogie : Rousseau s'efforce à chaque fois de montrer que ces théories du contrat de soumission se résument à fonder le droit sur la force, alors que la force ne fait pas droit. Il est important de souligner ce point car Hobbes, qui rejette l'idée de Loi naturelle et fonde la société sur le seul pouvoir politique positif, conserve à la force une vraie légitimité et une légitimité fondée sur la nature. En ce sens, chez Hobbes la société positive reste fondée sur le Droit naturel : ce droit illimité qui appartient par nature à tous est abandonné au seul chef au terme du contrat social et ce chef reste donc dans l'état de nature et exerce son Droit naturel, c'est-à-dire sa force supérieure. C'est pourquoi chez Hobbes, une insurrection, même si elle 
est menée par un groupe de malfaiteurs, si elle surpasse la force du chef, est légitime, fondée sur le Droit naturel. En conservant à la force une légitimité, Hobbes n'a pas complètement renoncé au Droit naturel. Rousseau va plus loin que Hobbes, et dès le premier paragraphe il s'attache à expulser le Droit naturel de la force en lui enlevant son caractère juridique pour ne lui laisser que son caractère naturel, c'est-à-dire la force, qui est simplement un fait physique :

La force est une puissance physique [...], la force ne fait pas droit, on n'est obligé d'obéir qu'aux puissances légitimes. (Rousseau, 1964c, I, ch.2)

Ainsi, non seulement Rousseau refuse la notion de Droit naturel traditionnelle mais il déracine même le dernier soupçon de nature juridique qui pouvait rester dans l'idée d'une force légitime. Sur une terre aussi ravagée, il sera difficile de faire germer quelque nouvelle pousse de la Loi naturelle.

La force ne fait pas droit, et pourtant c'est bien de la force que surgit, au chapitre six, la légitimité, la loi. Dans ce célèbre chapitre sur « Le pacte social ", on voit se constituer un peuple à partir d'une multitude, et cette constitution ne fait jamais appel à la Loi naturelle, mais ne cesse de parler de la force. Le passage de l'éparpillement des individus au « moi commun» est présenté comme un problème de pure physique : comment résister à une force supérieure à chaque force séparément qu'elle menace de détruire ? La réponse est toute physique : il faut unifier les forces en additionnant les quantités et en unifiant les directions, ce qui fera de toutes les forces une seule force. Galilée ou Newton avait habitués à cet usage algébrique des valeurs physiques. Solution physique qui soulève un nouveau problème, cette fois anthropologique: la liberté naturelle n'étant rien d'autre que l'usage de sa propre force, en l'agglomérant aux autres et en l'unifiant avec le mouvement général, on en perd l'usage, et on cesse donc d'être libre. La réponse à ce problème anthropologique est politique : en établissant une réciprocité absolue entre les associés, on bénéficie de la force supérieure de tous.

Cette solution est unique, elle ne répond pas à une loi naturelle préalable mais à la nature de l'acte, c'est-à-dire à l'essence de la politique. La politique n'existe pas avant la politique, mais à partir du moment où elle existe, elle a une nature propre:

Les clauses de ce contrat sont [...] déterminées par la nature de l'acte, [...] la moindre modification les rendraient vaines.

On pourrait dire la même chose d'une définition géométrique: il n'existe pas de triangle dans la nature mais à partir du moment où l'on trace 
un triangle sur le papier, ses trois angles valent deux angles droits, si ce n'est pas le cas, ce n'est pas un triangle, car cela ne correspond pas à la nature du triangle.

Le contrat étant établi, l'homme acquiert alors ses facultés, il devient citoyen, donc intelligent, de sorte que le contrat est en même temps une révolution anthropologique, il est « cet instant heureux [...] qui d'un animal stupide et borné, fit un être intelligent et un homme » (idem, I, ch.7).

Après l'établissement du Contrat, l'homme est donc devenu raisonnable, va-t-il présent se tourner vers la Loi naturelle, qu'il pourra enfin reconnaître ? Le Discours sur l'origine de l'inégalité avait écarté la Loi naturelle à cause des lumières bornées de l'homme naturel ; l'homme politique, désormais éclairé devrait y avoir accès. Comme on sait, la question de la loi, question posée par la vie commune que le Contrat a établie, cette question de la loi sera résolue par un « législateur » qui, à aucun moment, ne se tournera vers une quelconque Loi naturelle.

Il ne s'agit aujourd'hui de réfléchir sur cet énigmatique " législateur " mais seulement de voir si l'idée de Loi naturelle est compatible avec les activités qu'on lui assigne. Il aurait été facile de faire un législateur " profond métaphysicien » connaisseur de la Loi naturelle et qui aurait eu pour tâche de la concrétiser, en donnant au peuple une loi conforme à la nature humaine et à la justice éternelle. C'est tout le contraire, c'est exactement le contraire, que Rousseau propose : le législateur, loin de prendre en compte la nature humaine a pour tâche essentielle de la détruire et de mettre autre chose à sa place, une humanité artificielle :

Celui qui ose entreprendre d'instituer un peuple doit se sentir en état de changer [...] la nature humaine, [...] de substituer une existence partielle et morale à l'existence physique et indépendante que nous avons tous reçu de la nature [...]. Plus ces forces naturelles sont mortes et anéanties, [...] plus aussi l'institution est solide et parfaite.

Emile reprend presque mot pour mot cette idée et affirme que «les bonnes institutions sociales sont celles qui savent le mieux dénaturer l'homme » (Rousseau, 1969, I, p.249). On voit mal comment la Loi naturelle pourrait s'inviter à cette table où la nature est si radicalement desservie.

Mais poursuivons encore : au chapitre XII du livre II, Rousseau présente la division des lois : lois politiques, lois civiles, lois pénales, puis il ajoute une quatrième qu'il présente malignement en usant des mots généralement réservés à la Loi naturelle : une loi qui est au-dessus de toutes, qui est gravée dans les cœurs, qui domine toutes les lois : "A ces trois sortes de lois, il s'en joint une quatrième, la plus importante de toutes, qui ne se grave ni sur le marbre ni sur l'airain, mais dans le cœur des citoyens ». Mais cette loi n'est pas la Loi naturelle, c'est l'opinion publique, c'est-à-dire le peuple em- 
pirique, enfoncé dans son histoire et dans ses histoires, qui change lentement au gré des événements, qu'on ne doit pas bousculer mais qu'on modifie avec prudence et lenteur, tout le contraire d'une loi éternelle et universelle: " Je parle des mœurs, des coutumes et surtout de l'opinion; partie inconnue de nos politiques mais de laquelle dépend le succès de toutes les autres ; partie dont le législateur s'occupe en secret. » Ainsi la loi qui coiffe toutes les lois est plus encore que positive, elle est factuelle, irrationnelle, imprévisible, et en même temps indéracinable et solide, car elle était déjà là avant la loi et la loi doit s'y conformer sous peine d'échec. L'opinion a bien tous les caractères de la Loi naturelle, elle est préalable, supérieure, inscrite dans les cœurs, et elle en est la parfaite antithèse, ce n'est certainement pas un hasard.

Par son contenu, la législation du peuple exclut donc la Loi naturelle, mais elle l'exclut aussi par sa structure : je veux parler de la clef de voûte de la politique Rousseauiste, la volonté générale, le pacte fondamental. Le point central est l'affirmation selon laquelle aucune loi absolue ne s'impose au souverain, celui-ci étant le producteur des lois, il peut abolir ce qu'il a décidé, et même le contrat social n'a pas de force absolue pour le peuple souverain : « Il est contre la nature du corps politique que le souverain s'impose une loi qu'il ne puisse enfreindre. [...] Il ne peut y avoir nulle espèce de loi fondamentale obligatoire pour le corps du peuple, pas même le contrat social ॥ (Rousseau, 1964c, I, ch.7).

" Nulle espèce de loi fondamentale", on ne saurait être plus radical : la loi est le fait du peuple politique, il la fait et il la défait selon sa volonté. On peut remarquer, au passage, que Rousseau emploie dans cette phrase l'expression " la nature du corps politique " qui désigne ici encore l'essence de la politique, et la nature du corps politique ne signifie pas la nature dans le corps politique, pas plus que la nature du triangle ne signifie que les triangles poussent sur les arbres. C'est en ce sens, aussi, qu'il parle plus loin de ce qui est « conforme à l'ordre [...] par la nature des choses et indépendamment des conventions humaines " (idem, II, ch.6). Dans ce passage qui traite de la justice en soi, il remarque que cette " justice universelle », qui vient de "la raison seule", n'a aucune force pour sanctionner, elle est donc "vaine parmi les hommes », et même pire : dans la nature, c'est le méchant qui est récompensé et il faut des lois politiques pour rétablir les choses. Autrement dit, cette justice universelle n'a pas de valeur juridique, ce n'est pas une loi, c'est un principe moral mais non politique:

Faute de sanction naturelle, les lois de justice sont vaines parmi les hommes, elles ne font que le bien du méchant et le mal du juste. [...] Il faut donc des conventions et des lois pour unir les droits aux devoirs. 
L'affaire étant entendue, Rousseau balaye alors d'un revers de main la Loi naturelle et la jette dans la corbeille des "idées métaphysiques " qui n'ont aucun intérêt théorique pour qui veut comprendre la loi politique : "Quand vous aurez dit ce qu'est une loi de la nature, on n'en saura pas mieux ce que c'est que la loi de l'Etat ".

\section{Examen de quelques usages de la Loi naturelle}

Comme on sait, plusieurs lecteurs de Rousseau ont voulu aller plus loin que cette simple lecture, et en fouillant les textes, ils ont débusqué des formules assez discordantes avec ce que je viens de lire. Dans ces formules, il est question de Loi naturelle, et même de la supériorité de certaines lois sur les lois positives. Je propose d'examiner les principales affirmations de Rousseau que ces lecteurs citent volontiers pour montrer son attachement supposé au Droit naturel.

\section{Loi naturelle comme ordre de la nature}

Dans de nombreux cas, l'expression "Loi naturelle » désigne l'ordre de la nature, c'est-à-dire les lois physiques du mouvement, des êtres vivants. Il y a bien un ordre de la nature, voulu par Dieu, cela n'est pas douteux puisque Dieu aime l'ordre, ${ }^{7}$ mais cet ordre naturel n'a aucune forme juridique. Par exemple, dans le Discours sur l'origine de l'inégalité, Rousseau évoque l'émeute qui étrangle le tyran, et il renvoie dos à dos le tyran et les émeutiers, disant que c'est une question de pure force, que le droit n'y prend aucune part, et qu'on reste dans « l'ordre naturel ${ }^{8}{ }^{8}$ c'est dire si la Loi naturelle comprise somme ordre de la nature ne peut en aucun cas justifier l'idée d'un attachement au jusnaturalisme. Que cet ordre naturel soit suffisant pour « le sage », et que « les lois éternelles de la nature et de l'ordre [...] tiennent lieu de loi positive au sage ", comme on peut le lire dans Emile (Rousseau, 1969, p.857), ne prouve rien. Car le sage, c'est précisément celui qui n'a pas besoin de lois car il reste à sa place, en lui-même, aux frontières de la société, n'écoutant que son cœur et sa raison, et ignorant les passions

7 "La bonté de Dieu est l'amour de l'ordre » (Rousseau, 1969, IV, Vicaire savoyard).

8 "L'émeute qui finit par étrangler ou détrôner le sultan est un acte aussi juridique que ceux par lesquels il disposait la veille de la vie et des biens de ses sujets. La seule force le maintenait, la seule force le renverse ; toutes choses se passent ainsi selon l'ordre naturel » (Rousseau, 1964d, II, p.131). 
humaines qui, précisément, nécessitent les lois. Dire qu'un peuple de sages ou un peuple de Dieux n'auraient pas besoin de lois, cela ne plaide pas pour l'idée d'une Loi naturelle chez Rousseau. Le sage est libre au sens où l'homme sauvage est libre: "à la faveur des lois naturelles qui commandent à tous" (Rousseau, 1964e, VIII). Il s'agit toujours du déterminisme naturel. L'homme est libre dans le cadre du déterminisme naturel, des lois de la nature : on est plus proche de Spinoza que de Grotius et Pufendorf. Voilà pour le premier cas rencontré.

\section{Loi naturelle comme essence des choses}

Dans d'autres cas, Loi naturelle, loi de la nature, désignent la nature des choses, leur essence : soit leur essence réelle, soit leur essence rationnelle. Nature réelle : par exemple l'homme est sensible, libre. Nature rationnelle : un contrat d'esclavage est nul car un contrat suppose des contractants libres. En ce sens, la nature du peuple est l'égalité et la réciprocité des droits, c'est pourquoi toute convention politique qui supprime l'égalité et la réciprocité (le contrat de soumission) est une convention nulle, par la nature des choses. C'est en ce sens que Rousseau évoque des Lois naturelles, des lois de nature, qui dépassent les conventions. Ces lois de nature qui s'imposent au droit positif ne sont ni juridiques ni morales, elles sont logiques : quand Rousseau refuse le contrat d'esclavage ou le contrat de soumission, il ne disserte pas sur la justice ou la morale, il montre la contradiction conceptuelle qu'ils recèlent. Un contrat ne peut se faire qu'entre hommes, on ne fait pas de contrat avec les choses ou les bêtes ; un contrat d'esclavage qui ôte la liberté d'un des contractants annule l'homme et le contrat s'annule.

C'est en ce sens que Rousseau dit aux Polonais que la nature du peuple exige l'unanimité à sa formation, personne ne pouvant être forcé à rejoindre une association au nom d'une majorité, car la notion de majorité suppose que l'association est déjà formée et que tous y ont déjà consenti. Au commencement, seuls les volontaires peuvent être membres de l'association, d'où la loi d'unanimité qui est une sorte de tautologie, un jugement logique et non juridique :

Par le Droit naturel des sociétés, l'unanimité a été requise pour la formation du corps politique et pour les lois fondamentales qui tiennent à son existence. (Rousseau, 1964f, ch.9)

C'est encore dans ce sens qu'il parle de Loi naturelle dans les Lettres de la montagne: 
Par cette condition de liberté [...] toutes sortes d'engagements ne sont pas valides [...]. On doit prouver qu'il est convenable aux hommes et qu'il n'a rien de contraire aux Lois naturelles. Car il n'est pas plus permis d'enfreindre les Lois naturelles par le Contrat social qu'il n'est permis d'enfreindre les lois positives par des contrats particuliers, et ce n'est que par ces mêmes lois qu'existe la liberté qui donne force à l'engagement.

Dans ce texte, Rousseau explique : «Toutes sortes d'engagements ne sont pas valides " : le contrat social n'est pas un accord arbitraire factuel, il a une loi d'essence qui enveloppe la nature humaine, c'est-à-dire la liberté. Une convention entre les hommes est nulle si elle abolit ou néglige la liberté des hommes car l'engagement sans liberté n'est rien, il est sans force ; dire cela n'est en rien une façon d'accepter la Loi naturelle des jusnaturalistes. Rousseau dit ensuite que la liberté « existe par ces mêmes lois ", on voit mal en quoi la Loi naturelle juridique ferait exister la liberté, que serait une loi qui ordonnerait aux hommes d'être libres ? C'est absurde, il s'agit d'une loi au sens d'une détermination de l'essence humaine.

\section{Loi naturelle comme loi morale}

Dans un troisième cas, la Loi naturelle porte sur la morale, dont la pitié est la source naturelle, et que la conscience, " instinct divin ", établit après l'avènement de la raison. Concernant la morale, il est possible de parler d'une morale en deux temps, une morale naturelle d'abord, sous forme de pitié instinctive, et une morale raisonnable ensuite. Le deuxième moment élève le premier au niveau proprement humain, le fait passer de la sensibilité à l'imagination et la raison.

Mais la morale n'est pas la politique. Les lois morales ne sont pas sanctionnées parmi les hommes, elles ne sont fondées que sur l'espérance raisonnable d'un monde ordonné, équilibré, qui permet d'espérer que les biens et les maux seront compensés dans un autre monde (c'est la leçon du Vicaire savoyard). L'assimilation de la loi morale à la loi politique ne semble pas autorisée. C'est au sens moral que Rousseau parle dans Du Contrat social de « Droit naturel divin » :

[La religion] sans temples, sans autels, sans rites, bornée au culte purement intérieur du Dieu suprême et aux devoirs éternels de la morale est la pure et simple religion de l'Evangile, le vrai théisme, et ce qu'on peut appeler le Droit naturel divin. (Rousseau, 1964c, I, ch.8, p.464)

On retrouve encore la Loi naturelle morale dans un brouillon sur L'état de guerre (Rousseau, 1964g, p.602), et ce passage est quelquefois cité à tort en témoignage de la présence d'une Loi naturelle chez Rousseau, alors qu'il s'agit simplement de la pitié : 
Si la Loi naturelle n'était écrite que dans la raison humaine, elle serait peu capable de diriger la plupart de nos actes, mais elle est encore gravée dans le cœur de l'homme [...]. C'est là qu'elle lui crie qu'il ne lui est pas permis de sacrifier la vie de ses semblables qu'à la conservation de la sienne.

Si on se souvient que la base de la loi est l'égalité et la réciprocité, on voit bien que cette loi qui interdit de faire du mal sans utilité n'a rien à voir avec la loi et qu'elle ne l'inspire à aucun titre.

Si on doute de la différence de nature qui existe chez Rousseau entre la loi morale et la loi juridique, on n'a qu'à se souvenir que, pour connaître la Loi naturelle juridique, il faudrait être " un profond métaphysicien », tandis que pur connaître la loi morale, c'est tout le contraire : "Grâce au ciel, nous voilà débarrassés de tout cet effrayant appareil de philosophie, nous pouvons être hommes sans être savants » (Rousseau, 1969, IV, p.601).

\section{Loi naturelle et " autorités "}

Enfin, pour terminer cette revue, on doit citer une lettre, dans laquelle Rousseau déclare très clairement que le souverain politique doit être soumis à trois autorités qui lui sont supérieures, et on a cru y voir un rapport entre la loi positive et la Loi naturelle. Voyons de plus près. Dans cette lettre du 15 octobre 1758 (Rousseau, 1934, IV, p.87-8), Rousseau répond aux accusations de hobbisme, en ce qu'il donnerait au pouvoir politique une légitimité arbitraire et absolue. Il répond qu'il existe "trois autorités supérieures " au contrat social. Il dit " autorités » et non « loi », on va voir pourquoi. Voici le texte :

J'en admets trois seulement. Premièrement l'autorité de Dieu, et puis celle de la Loi naturelle qui dérive de la constitution de l'homme, et puis celle de l'honneur plus forte sur un cœur honnête que tous les rois de la terre.[...] Non seulement indépendantes mais supérieures. Si jamais l'autorité souveraine pouvait être en conflit avec une des trois précédentes, il faudrait que la première cédât en cela.

Ces trois « autorités » peuvent elles constituer un Droit naturel, c'est-àdire une loi antérieure, supérieure et fondatrice du droit?

La première est l'autorité de Dieu, dire qu'elle est supérieure à tout ne coûte pas bien cher, Du Contrat social a déjà répondu à cette question en affirmant que si « toute justice vient de Dieu », il se trouve qu'on ne la reçoit pas de si haut et qu'il faut la fonder de tout autres bases, de sorte qu'en vérité la justice vient de la loi, et non la loi de la justice. L'autorité de Dieu, le Vicaire le montre bien, n'est pas juridique mais physique avec l'ordre du monde, métaphysique avec les compensations après la mort, et morale avec 
la conscience. Dieu ne donne pas une loi aux hommes, et quand le législateur s'attache à la leur donner, il n'attend aucune révélation, et même son usage de l'autorité divine relève plutôt du stratagème tactique, c'est une manière de briser les résistances et les méfiances. On ne peut donc identifier l'autorité de Dieu à une Loi naturelle juridique.

La deuxième est " la Loi naturelle qui dérive de la constitution de l'homme ». On retrouve le cas déjà rencontré concernant l'essence des choses : la liberté humaine est essentielle à la politique, la nier c'est nier la politique au profit de la force sauvage, en ce sens elle supérieure.

La troisième est morale, c'est " l'honneur " qui autorise la désobéissance. Il y a là, effectivement, un accroc par rapport au texte de Du Contrat social. Dans ce texte, celui qui désobéit, qui veut sortir du contrat, " on le forcera d'être libre "). Libre, c'est-à-dire respectueux du contrat qu'il a souscrit et qui a formé le souverain. A présent Rousseau dit que l'honneur prime sur le souverain. Mais le souverain n'est pas le même, ici ce n'est plus le peuple mais " les rois de la terre ", c'est donc un souverain non légitime. On trouve la même idée dans Emile : puisqu'il faut bien vivre avec de mauvaises lois et leur obéir, le mieux est d'être honnête, c'est une façon d'être inutile à cette politique là. Il ne s'agit pas d'opposer une Loi naturelle à la loi positive définie par le peuple souverain, mais d'opposer la conscience morale au "simulacre " des lois existantes. On peut s'étonner de ce texte qui autorise la désobéissance au nom de la morale, mais il ne s'agit pas, en tout cas d'une affirmation jusnaturaliste.

\section{La pitié et la Loi naturelle}

Parmi ceux qui décèlent la présence d'une Loi naturelle chez Rousseau, certains affirment qu'on peut étendre la Loi naturelle morale, la pitié, la conscience, à la Loi naturelle politique. Si on veut dire qu'il est possible, à partir des notions morales, de concevoir ce que pourrait être une Loi naturelle rousseauiste à laquelle Rousseau n'a pas songé, alors la formule est tout à fait acceptable : être rousseauiste, cela peut consister à produire des infidélités fécondes. On peut être rousseauiste en révisant Rousseau, comme Plotin est platonicien, comme Fichte est kantien, comme Marx est hégélien, comme Gramsci est marxiste. Mais on doit ajouter que dans le texte même de Rousseau, rien n'autorise le glissement de la morale à la politique. Il y a bien une morale dont la loi dépasse la volonté humaine et qui s'impose aux individus par le moyen de la sensibilité, de la raison ou de la conscien$\mathrm{ce}$, mais on ne trouve rien de tel dans le domaine de la politique. Dans le domaine politique, le droit, la justice, la loi, sont les effets consécutifs de la loi positive, c'est-à-dire de l'existence d'un peuple, et cela est posé en théo- 
rie comme en pratique par Rousseau. Aucune voix de Dieu ne s'impose au peuple, car il est lui-même, " la voix de Dieu " (Rousseau, 1964a, p.246). ${ }^{9}$

On peut, certes, s'étonner de ce que Rousseau soit idéaliste en morale et réaliste en politique, on peut affirmer qu'il y a là une difficulté, voire une contradiction : cette contradiction existe bien et elle constitue le "système " de Rousseau. Pour lui, ce n'est pas une contradiction, et il assume clairement ce hiatus entre morale et politique, comme le montre la fin de $\mathrm{Du} C \mathrm{Con}$ trat social. Rousseau affirme, en effet, que les Evangiles sont un guide sublime pour la morale, mais il considère en même temps qu'un peuple de chrétiens serait la perte de la république : c'est dire si la vertu morale et la vertu politique s'accordent mal ; on ne doit pas s'étonner, après cela qu'il existe une Loi naturelle morale et que toute loi politique soit positive et rien d'autre, ces deux mondes sont étanches. On peut tenter, à partir de Rousseau et contre lui, de réconcilier ces deux mondes et de renouveler le rousseauisme, mais ce faisant on sort du système. ${ }^{10}$

\section{Quelques problèmes théoriques}

Si donc on s'en tient à Rousseau et à son système, on doit, pour finir, examiner encore deux aspects.

\section{La preuve par Emile}

Le premier concerne encore le Droit naturel raisonné. Comme je l'ai indiqué, il existe une interprétation selon laquelle il y aurait un Droit naturel en deux temps, en deux figures.

1. Il existe une Loi naturelle indépendante, préalable, supérieure à la loi positive ; mais les hommes sont trop stupides, à l'origine, pour le comprendre ;

2. Quand les hommes ont développé leur raison grâce à la société qui a impulsé ce développement, ils pourraient donc enfin connaî-

9 Jean-Jacques Rousseau, Economie politique : "La voix du peuple est en effet la voix de Dieu » (Rousseau, 1964a, p.246)

10 Ainsi, André Charrak annonce clairement : "Il est cependant plus prudent de laisser de côté le problème d'un système de Rousseau pour examiner, etc. " (Charrak, 2002). De même, Ghislain Waterlot s'efforce de produire chez Rousseau une vision de la politique chrétienne dans une Europe réconciliée (Waterlot, 2004). Ces tentatives profondes et talentueuses montrent que le rousseauisme après Rousseau peut exister et on doit s'en réjouir. 
tre cette loi première, mais comme la société a, en même temps, corrompu leur cœur, ils sont incapables de sentir cette loi, et donc de la connaître.

Il y aurait donc deux rendez-vous manqués, le premier parce que l'homme sensible n'est pas raisonnable, le deuxième parce que l'homme raisonnable n'est plus sensible; ainsi la Loi naturelle reste inconnue d'abord pour une nature humaine non développée, et ensuite à cause d'une développement dénaturé.

Il est donc facile de concevoir qu'un homme qui se serait développé sans être dénaturé, serait très capable de reconnaître, après-coup cette Loi naturelle en attente.

Je crois qu'il existe un moyen simple et rapide de trancher cette question, c'est Emile. "L'homme abstrait » qui grandit dans ce texte est un sauvage destiné à devenir social sans être, pour autant, dénaturé. Il sera parfaitement développé par la société sans être corrompu par elle. Si donc la Loi naturelle existe selon Rousseau, notre élève, le petit Emile, parvenu à l'âge de devenir citoyen, devra nécessairement reconnaître la Loi naturelle. Or, que voyons-nous ? Quand Emile devient sociable, c'est-à-dire sexué, il découvre le monde humain, " il n'est plus seul ", et du même coup il découvre ses devoirs moraux : ses sens, son imagination et sa raison s'unissent pour lui indiquer la route du bien. Mais quand il s'agit de se marier, c'est-à-dire d'être époux et père, il doit se préparer à être citoyen et donc il doit connâ̂tre les principes de la politique. Si Rousseau concevait qu'il existe un droit naturel préalable et supérieur au droit, le gouverneur d'Emile s'attacherait à ce moment à le lui présenter; or c'est tout autre chose qu'il lui présente. Il lui présente les principes du pacte social et de la volonté générale, sans aucune référence à un Droit naturel. Finalement, après avoir visité les pays d'Europe, et devant le tableau navrant que présente la politique réelle, Emile ne sait que faire, alors le gouverneur change de ton et lui commande de vivre dans son pays de naissance, sous des « simulacres » de lois qui valent mieux que pas de loi du tout ; et pour se soustraire à cette corruption dans le cas où il serait appelé à servir le Prince, il ne lui conseille pas, aux commandes du pouvoir, d'appliquer quelque principe de Droit naturel, raisonné ou pas, il lui conseille d'être honnête, meilleure façon d'être inutile en politique. Le seul préservatif à la corruption politique est la morale et non la Loi naturelle, on retrouve les termes de la lettre qui affirme que l'honneur est plus fort que les rois de la terre.

Ainsi donc, pour un homme social raisonnable non dénaturé, la Loi naturelle n'est pas au programme, preuve qu'elle n'était pas dans la nature précédemment. 


\section{La Loi naturelle comme garantie du contrat social}

Deuxième point concernant le système. On a pu remarquer qu'il existe une difficulté : si Rousseau expulse le Droit naturel de son univers humain, comment peut-il concevoir un contrat social ? En effet, pour faire un contrat, les individus qui contractent s'engagent, c'est-à-dire qu'ils sont conscients qu'ils doivent tenir leur parole, rester fidèles à leur engagement dans la durée. Cette règle est donc préalable au contrat qui établit le droit, c'est une obligation légale qui fonde la loi, c'est une loi avant la loi : ce thème revient en permanence chez les jusnaturalistes, et même chez Hobbes.

En établissant la loi positive sur un contrat, Rousseau devrait donc supposer une Loi naturelle sous peine de contradiction. ${ }^{11}$

Ces arguments sont très forts. Mais on doit voir que chez Rousseau, le " contrat social " est tout le contraire d'un contrat. Structurellement, ce n'est pas un contrat : Althusser l'a démontré (Althusser, 1967), pour établir un contrat, il faut que les contractants existent avant le contrat, or ce n'est pas le cas puisque Rousseau dit que chacun fait un contrat avec tous, un " tous " qui n'existe pas avant le contrat puisque c'est le contrat qui crée l'association, qui fait exister le tous. Pour que chacun puisse faire un contrat avec tous il faut que le contrat qui fait exister le tous existe avant que chacun le contracte, ce qui est circulaire : le contrat doit exister avant le contrat pour pouvoir exister, etc. Du point de vue structurel ce n'est donc pas un contrat.

Mais ce n'est pas non plus un contrat du point de vue génétique. Dans la genèse du contrat rousseauiste, il n'y a pas de moment au cours duquel une décision est prise. Les individus se fondent en un peuple sous la pression de la peur de mourir, mais ils ne prennent aucune décision : "Le genre humain périrait s'il ne changeait sa manière d'être ". Rousseau décrit le passage de la multitude au peuple comme une mécanique de forces : «Ils n'ont plus d'autre moyen pour se conserver que de former une somme de forces » (Rousseau, 1964c, I, ch.6) ; et d'ailleurs il précise ensuite que c'est le contrat qui a donné aux hommes leur intelligence, leur raison, ce qui signifie qu'avant le contrat ils sont incapables de volonté, de décision, de réflexion : "D'un animal stupide et borné [il] fit un être intelligent et un homme " (idem, I, ch.8). Dénués de toutes les facultés qui auraient pu leur permettre de comprendre de ce qu'ils étaient en train de faire, les individus sont devenus peuple sans s'être jamais engagé à rien. Ce n'est pas la pen-

11 Cette difficulté soulevée par Vaughan est reprise en ces terme par Robert Derathé dans son ouvrage monumental, Rousseau et la science politique de son temps : " Si l'on supprime la Loi naturelle, le contrat social, privé de toute sanction morale, n'a plus d'autre garantie que la force " (Dérathé, 1995, p.159). 
sée qui constitue un peuple, c'est l'acte. Tous se sont trouvés engagés dans un mouvement nécessaire à leur survie, un mouvement par la force des choses que personne n'a voulu et que personne ne regrette, car il est, aprèscoup, désiré par tous, "béni » par chacun. On ne peut pas déduire les caractères du peuple à partir des caractères des individus car le peuple ne se déduit pas des individus, il émerge à partir d'eux et les dépasse en acte. Il n'est pas nécessaire que les individus connaissent les devoirs de l'engagement pour s'engager. La causalité chez Rousseau n'est pas hypothético-déductive mais hypothético-émergente (cf. Vargas, 2005, p.173-6).

Il y a pourtant une difficulté, la dernière. Je viens de dire que les individus apeurés et attroupés ne se sont pas engagé à être membres d'un peuple. Est-ce à dire que, ne s'étant pas engagés, ils peuvent se dégager quand il leur plaît? Pas du tout, s'ils veulent se soustraire à la loi, « on les forcera d'être libres ", c'est-à-dire de se plier à la volonté générale. Au nom de quoi pourra-t-on les forcer? Au nom, nous dit Rousseau, d'une convention " tacite ", celle de tenir ses engagements. Voici donc la Loi naturelle qui revient, comme si elle était présente mais muette, " tacite » :

Afin que le pacte ne social ne soit pas un vain formulaire, il renferme tacitement cet engagement [...] que quiconque refusera d'obéir à la volonté générale y sera contraint par tout le corps. (Rousseau, 1964c, I, ch.6)

Pour y voir clair, le mieux est de voir ce que Rousseau entend par ce mot " tacite ". Par chance, il définit ce qu'il entend par là dans le manuscrit de Genève, et donne un exemple qui montre que " tacite " ne signifie pas un rapport volontaire muet mais une évidence sans laquelle les choses deviennent absurdes, on est plus près de la logique, de l'essence des choses que d'un commandement. Rousseau utilise cet idée pour rejeter un contrat de soumission, même si ce contrat était accepté par le peuple : le peuple alors serait fou car un tel contrat serait contraire à la nature même de l'accord. Voici l'explication :

Pour éclaircir cette vérité, il faut bien remarquer que ceux qui prétendent qu'une promesse gratuite oblige rigoureusement le promettant, distinguent pourtant avec soin les promesses purement gratuites de celles qui renferment quelques conditions tacites mais évidentes [...] Un peuple qui se choisit un ou plusieurs chefs et promet de leur obéir suppose évidemment qu'ils ne feront de sa liberté qu'il leur aliène qu'un usage avantageux pour lui-même, sans quoi ce peuple étant insensé ses engagements seraient nuls. (Rousseau, 1964b, I, ch.2, p.315)

On voit bien ici que la condition tacite ne porte pas sur la volonté du chef qui est choisi mais sur l'essence même de l'acte, c'est une loi d'essence et en politique les lois d'essence apparaissent à tel ou tel moment com- 
me des actes : refus d'obéir au Prince, obligation d'obéir à la loi, parce qu'en politique la loi d'essence se réalise dans le temps, mais elle reste liée à la chose en sa nature propre. Ce que confirme la fin du paragraphe : "qui sans cela seraient absurdes, tyranniques et sujets aux plus énormes abus. »

Faut-il conclure que Rousseau est positiviste comme Hobbes, et accepter par là l'accusation de "totalitarisme "? Je crois que pour dégager Rousseau de cette infamie, il n'est pas nécessaire de réactiver une loi naturelle qu'il a continuellement rejetée. L'affirmation que la loi fonde la justice et qu'aucune loi ne peut être injuste, est certes une affirmation positiviste ; mais on doit voir que chez Rousseau la loi, ce qui mérite être appelé « loi ", c'est la volonté du peuple, c'est la règle que le peuple s'applique à lui-même, tout le reste n'est qu'un simulacre de loi.

Il faut mesurer toute la portée théorique du concept de peuple chez Rousseau : ce n'est pas une notion descriptive, c'est un concept opérationnel, explicatif, c'est le concept à partir duquel le mot " politique " prend un sens, à partir duquel la force devient légitime, etc. La loi n'a pas besoin de garantie supérieure parce qu'avant le peuple il n'y a rien et il n'y a rien non plus au-dessus de lui : la loi de nature, même si elle existait, ne serait pas audessus du peuple puisque c'est par le peuple que l'homme acquiert sa nature.

Mais la loi de nature n'a jamais exister et il n'est pas certain qu'un vrai peuple ait commencé à existé, depuis les Spartiates et les Romains ont perdu leur vertu antique.

VARGAS, Yves. Rousseau and natural right. Trans/Form/Ação, (São Paulo), v.31(1), 2008, p.25-52.

- SUMMARY: It is a mistake to grant Rousseau a favourable position to natural right. A careful reading of texts explains this author's complex, more radical fashion of refusing to make any concession to this concept.

- KEYWORDS: Rousseau, Right Natural, Positivism, Natural law.

\section{Références bibliographiques}

ALTHUSSER, Louis. "Sur le Contrat Social (les décalages)». Revue Cahiers pour l'analyse n.5. Paris : Cercle d'Epistemologie de l'École Normale Superieure, 1967.

BURLAMAQUI, J.-J. Principes de Droit naturel. Caen : Presse Universitaire de Caen, 1989. 
CHARRAK, André. « Du Droit naturel au Droit naturel raisonné ». Revue Cahiers philosophiques de Strasbourg, n.13. Strasbourg : Presse Universitaire de Strasbourg, 2002

DERATHE, Robert. Rousseau et la science politique de son temps. Paris : Vrin, 1995.

GROTIUS, H. Droit de la guerre et de la paix. Caen : Presse Universitaire de Caen, 1984.

LOCKE, J. Second traité du gouvernement civil. Paris : Garnier-Flammarion, 1992.

ROUSSEAU, J.J. Oeuvres complètes. 5 tomes. Collection Pléiade. Paris : Gallimard, 1959-1995 1934.

Correspondance générale, t.IV. Anot. Théophile Dufour. Paris: Colin,

_. Economie politique. In : OC, t III, 1964a.

. Manuscrit de Genève. In : OC, t.III, 1964b.

. Du Contrat social. In : OC, t.III, 1964c.

- Discours sur l'origine de l'inégalité. In : OC, t.III, 1964d.

. Lettres de la montagne. In : OC, t.III, 1964e.

. Considérations sur le gouvernement de Pologne. In : OC, t.III, $1964 f$.

. Fragments politiques. OC, t.III, 1964g.

Emile. In : OC, t.IV, 1969.

VARGAS, Yves. Les promenades matérialistes de Jean-Jacques Rousseau. Paris : Le temps des cerises, 2005.

WATERLOT, Ghislain. Rousseau, religion et politique. Paris : PUF, 2004. 